\title{
Approaches for estimating minimal clinically important differences in systemic lupus erythematosus
}

\author{
Sharan K Rai ${ }^{1,2}$, Jinoos Yazdany ${ }^{3}$, Paul R Fortin ${ }^{4}$ and J Antonio Aviña-Zubieta ${ }^{1,5^{*}}$
}

\begin{abstract}
A minimal clinically important difference (MCID) is an important concept used to determine whether a medical intervention improves perceived outcomes in patients. Prior to the introduction of the concept in 1989, studies focused primarily on statistical significance. As most recent clinical trials in systemic lupus erythematosus (SLE) have failed to show significant effects, determining a clinically relevant threshold for outcome scores (that is, the MCID) of existing instruments may be critical for conducting and interpreting meaningful clinical trials as well as for facilitating the establishment of treatment recommendations for patients. To that effect, methods to determine the MCID can be divided into two well-defined categories: distribution-based and anchor-based approaches. Distribution-based approaches are based on statistical characteristics of the obtained samples.

There are various methods within the distribution-based approach, including the standard error of measurement, the standard deviation, the effect size, the minimal detectable change, the reliable change index, and the standardized response mean. Anchor-based approaches compare the change in a patient-reported outcome to a second, external measure of change (that is, one that is more clearly understood, such as a global assessment), which serves as the anchor. Finally, the Delphi technique can be applied as an adjunct to defining a clinically important difference. Despite an abundance of methods reported in the literature, little work in MCID estimation has been done in the context of SLE. As the MCID can help determine the effect of a given therapy on a patient and add meaning to statistical inferences made in clinical research, we believe there ought to be renewed focus on this area. Here, we provide an update on the use of MCIDs in clinical research, review some of the work done in this area in SLE, and propose an agenda for future research.
\end{abstract}

\section{Introduction}

A minimal clinically important difference (MCID) is an important concept used to determine whether a medical intervention improves perceived outcomes in patients. Prior to the introduction of the concept in 1989, studies focused primarily on statistical significance [1]. As clinicians, investigators, and policy-makers are becoming increasingly interested in incorporating patients' attitudes, priorities, and perspectives on disease in the longitudinal evaluation of novel intervention strategies, questionnaires assessing health-related quality of life (HR-QOL) and perceived health status are gaining widespread use. However,

\footnotetext{
*Correspondence: azubieta@arthritisresearch.ca

${ }^{1}$ Arthritis Research Canada, 5591 No. 3 Road, Richmond, BC V6X 2C7, Canada ${ }^{5}$ Division of Rheumatology, Department of Medicine, University of British Columbia, Suite 802 - 1200 Burrard Street, Vancouver, BC V6Z 2C7, Canada Full list of author information is available at the end of the article
}

despite the abundance of such instruments, their interpretability poses a challenge to investigators. MCID directly addresses the limitations of examining statistical significance in isolation, particularly the possibility that studies may find statistical relationships that do not have clinical importance to patients, clinicians, or policymakers.

Systemic lupus erythematosus (SLE) is a chronic, multisystem autoimmune disease that illustrates some of the challenges posed by defining and measuring MCIDs. SLE is a heterogeneous disease with a wide variety of symptoms in individual patients and across the population. Furthermore, SLE is characterized by periods of low disease activity alternating with periods of higher disease activity, a pattern that directly impacts the patient's quality of life [2]. However, outcome measures that capture

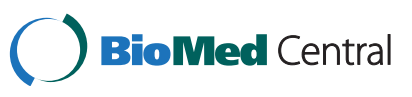


the complexity of SLE and adequately reflect the broad array of symptoms and signs have been challenging to both develop and apply, and limited work has been done to define MCIDs for existing patient-reported measures [3]. Although there has been work applying generic health status instruments in SLE (for example, the Medical Outcomes Study 36-Item Short Form (SF-36), for which an improvement of 2.5 points has been defined as the MCID in SLE [4, 5]), these tools are known to have relatively poor responsiveness in SLE $[6,7]$. Thus, there is a need to bring new focus and methodology to MCID measurement in SLE. Here, we provide an update on the use of MCIDs in clinical research, review some of the work done in this area in SLE, and propose an agenda for future research.

\section{Defining a clinically meaningful difference}

The MCID has been proposed as the 'smallest difference in score in the domain of interest which patients perceive as beneficial and which would mandate, in the absence of troublesome side effects and excessive cost, a change in the patient's management' [1]. The MCID therefore constitutes a threshold for outcome scores (either patient-reported or physician-measured) over which a patient or physician would consider a given change in score to be meaningful and worthwhile, which is critical for conducting clinical trials in SLE as well as for facilitating the establishment of treatment recommendations for patients $[2,8]$.

\section{Minimal clinically important difference in the context of systemic lupus erythematosus}

The Belimumab in Subjects With Systemic Lupus Erythematosus (BLISS)-52 [9] and BLISS-76 [10] trials of belimumab (the first drug approved for SLE in over 50 years) employed the SLE Responder Index (SRI), the first composite measure of SLE disease activity that incorporates criteria from three different validated indices: the Safety of Estrogens in Lupus Erythematosus - National Assessment-SLE Disease Activity Index (SELENA-SLEDAI), a Physician Global Assessment, and the British Isles Lupus Assessment Group (BILAG) instrument [11]. This composite index provides a more comprehensive assessment of SLE disease activity because it uses several instruments simultaneously, thus leveraging the relative advantages and disadvantages of various available indices [11]. In addition to the SRI, the Physical Component Summary (PCS) of the SF-36, a generic instrument for measurement of HR-QOL that has been validated for use in SLE clinical trials, was used to assess change in HR-QOL following treatment with belimumab $[9,10]$. Results of the trial showed significant improvement in SF-36 scores across both belimumab groups (1 and $10 \mathrm{mg} / \mathrm{kg}$ ) at week 52, which correlated with an SRI response (as compared with non-responders)
[9]. However, as the SF-36 is not a measure of disease activity, it was not included in the SRI [12].

Unlike the BLISS trials, many other recent trials - for example, the Exploratory Phase II/III SLE Evaluation of Rituximab (EXPLORER) [13], the Lupus Nephritis Assessment with Rituximab (LUNAR) [14], and the abatacept [15] trials - have all reported non-statistically significant results. In the case of rituximab, this is especially disappointing in light of several smaller uncontrolled trials that suggested potential efficacy in SLE [16-19]. Thus, the manner in which a response is defined holds the potential to determine whether a clinical trial is deemed a success or a failure [20]. This has been demonstrated in the context of lupus nephritis, in which Wofsy and colleagues [20] aimed to determine which response criteria are most sensitive to differences among treatment groups.

Thus, as several clinical trials in SLE to date have failed to show significant effects, defining the MCID of existing instruments (as well as composite indices, such as the SRI) may be critical for the conduct of interpretable and meaningful clinical trials in SLE, as it will help determine the effect of a given therapy as well as assist with appropriate design of clinical trials by informing the estimation of effect size, thus facilitating sample size calculation.

\section{Perspectives on the minimal clinically important difference}

The MCID can be defined from the perspective of the patient (or the patient's proxy, such as a caretaker or partner), health-care professionals, or researchers [21, 22]. For example, the patient may consider a meaningful difference to be one that results in a reduction of symptoms or an improvement in function, thus allowing him or her to perform an essential task or to perform tasks more efficiently (for example, with less pain), but this would not necessarily take into account an intervention's impact on survival or damage [21]. Conversely, a physician may define a meaningful difference to be a change in treatment or disease prognosis [23]. Additionally, the MCID can be further defined from the perspective of society, which would define a meaningful change as one that allows a patient to return to employment, or of the payers (for example, an insurance company), who would define a meaningful change as one that produces a claim closure [22]. Given the diversity of available perspectives, definitions of the MCID may (and likely will) be discordant.

The MCID may also be defined at either the individual or group level (and will vary accordingly). Inferences made at the group level can inform comparisons between different treatments or decisions regarding public policy; conversely, inferences made at the individual level can inform individual clinical treatment decisions [23]. Furthermore, when considering the magnitude necessary for a change to be considered important, larger changes may be required 
at the individual level, whereas relatively smaller changes may be interpreted as clinically important when considered at the group level [23, 24].

\section{Health-related quality of life}

The discordance between physician and patient perspectives raises the question of who ought to decide what constitutes a clinically meaningful change. Patients' perceptions of clinically worthwhile changes are influenced by their health status at baseline as well as their expectations, needs, and goals [21]. Conversely, the clinician's judgment draws upon previous knowledge and experiences, consideration of things that could be treated, and an understanding of physiologic findings that may not be symptomatic to the patient [21]. Thus, it has been suggested that for measures of physical function and quality of life, responsiveness should be based on the subject's perception of meaningful change but that for measures of impairment or disease activity, the physician may provide the best judgment [21].

To that effect, various international and multidisciplinary bodies (for example, the Outcome Measures in Rheumatology group) have developed a core set of outcome domains for rheumatic diseases (as had originally been done for rheumatoid arthritis clinical trials) [25, 26]. Specifically, they have recommended that the following constitute a core set of domains for SLE clinical trials: disease activity, HR-QOL, adverse events, and cumulative organ damage $[25,26]$. Whether some of these domains should be combined into a composite index (as in the SRI) or evaluated individually warrants further investigation.

The importance of including HR-QOL as a core domain cannot be overstated, as it would incorporate the patient's perspective of the impact of therapy on various physical, social, and psychological aspects of their health. For example, although a new therapy might show a clinically relevant improvement in disease activity as measured by available indices, this improvement may be contrasted by a clinically meaningful worsening in the patient's HR-QOL (for example, due to side effects). The converse may also be true: subjective improvements (for example, fatigue, an extremely disabling symptom among patients with SLE) may not be captured by a disease activity index. In this case, capturing clinically meaningful changes in fatigue level could provide great insight into the development and acceptance of new therapeutic agents. Indeed, a trial of the efficacy of abatacept reported that although the study's primary and secondary endpoints were not met, treatment effects were seen in certain exploratory patient-reported measures, such as in the SF-36, problems with sleep, and fatigue [15].

\section{Methods to determine the minimal clinically important difference}

There are various methods to calculate the MCID and each has relative advantages and disadvantages. An extensive review of available methods was published by Wells and colleagues [27], who classified them into nine different approaches. Another review proposed three distinct categories of approaches for defining the MCID: distribution-based (using statistical descriptions of the population), opinionbased (relying upon experts), and predictive/data-driven (using sequential hypothesis formation and testing) [28]. Overall, regardless of the larger framework employed, methods to determine MCIDs can be divided into two well-defined categories: distribution-based and anchorbased approaches [29]. However, despite this dichotomous classification, distribution-based methods confer the most use when they are applied together with a meaningful external anchor [30].

\section{Anchor-based methods}

Anchor-based approaches compare the change in a patientreported outcome with a second, external measure of change, which serves as the anchor [29]. Given the large selection of external criteria, this approach can be quite varied [31]. The anchor can be either an objective (for example, medication use or health-care utilization) or subjective (for example, patient self-report of improvement or worsening) measure; however, given the limited availability of acceptable objective assessments, few studies have employed an objective anchor [32, 33]. Instead, anchorbased methods generally rely on the use of a subjective assessment (most commonly a global assessment) [32]. Importantly, these anchor-based methods have the advantage of linking the change in a given score to the patient's perspective (which is captured by the anchor) [23].

According to a comprehensive review by Copay and colleagues [32], four variations of the anchor-based approach can be described: (a) the 'within-patients' score change, (b) the 'between-patients' score change, (c) the sensitivity- and specificity-based approach, and (d) the social comparison approach. Another extensive review, by Crosby and colleagues [23], summarized various anchor-based methods for determining individual change according to crosssectional versus longitudinal methods, with longitudinal methods being more linked with change and thus conferring a benefit over cross-sectional methods. To that effect, employing a longitudinal approach is of particular benefit in SLE, which is characterized by fluctuating status due to flares and remissions.

In addition to determining the responsiveness of the SLE Activity Questionnaire (SLAQ) by using the standardized response mean (SRM), the aforementioned study by Yazdany and colleagues [34] further examined the responsiveness of the SLAQ among a large observational cohort of SLE patients by employing clinically relevant and validated patient assessments of disease activity and health status (for example, the SF-36 Physical Functioning subscale) as the anchors. SLAQ scores were found to 
correlate strongly with these other health instruments (that is, the anchors), with the exception of the ShortForm 12 PCS [34].

\section{Limitations of the anchor-based methods}

First, the application of different anchors or anchor types may produce different values of the MCID [32], although this is not unlike the distribution-based methods in which different statistical approaches will also produce a variety of MCID values. Additional limitations include a potential discordance of defined MCID values based on whether data collection of the anchor was prospective versus retrospective [35], the possibility that the MCID as determined by anchor-based methods falls within the instrument's random variation [23], and the susceptibility of some ratings to recall bias [23] (although perhaps this may be alleviated by considering the necessity or wish for change in medication at a given time point, rather than the change over time, which warrants further research).

\section{Distribution-based methods}

Distribution-based interpretations are based on statistical characteristics of the obtained samples [29]. There are various methods within the distribution-based approach, including the standard error of measurement (SEM), the standard deviation, the effect size, the minimal detectable change, the reliable change index (RCI), and the SRM.

\section{Standard error of measurement}

The SEM is defined as the variation in patient-reported outcome scores attributed to instrument unreliability, in which a change smaller than the calculated SEM is likely due to measurement error rather than a true change [32]. Thus, the SEM is considered to be a characteristic of the measure, not the sample [36]. To define the MCID, threshold values of 1 SEM, 1.96 SEM, and 2.77 SEM have been suggested [36-38]. To illustrate, the MCID can be defined by using the SEM of changes in disease activity scores of SLE patients who have stable disease (that is, patients rated as having no change in disease between consecutive visits) [39]. This was done in a recent study that determined the MCID of validated measures of SLE disease activity in childhood-onset SLE [39]. The MCID was based on both the 1-SEM criterion (which makes the assumption that meaningful improvement or worsening has occurred if the change is plus or minus 1 SEM, respectively) and a more strict criterion (that is, \pm 1.645 SEM) [39]. A tighter confidence interval resulted in a more accurate detection of patients with stable disease versus those who experienced clinically important change [39].

\section{Standard deviation}

Another measure of variability is the standard deviation [32], defined as the variation among a group of scores, for which 0.5 standard deviations has been suggested to correspond to the MCID in a number of studies [40]. An example of this method is provided in a study by Katz and colleagues [41], in which changes in valued life activity scores from baseline to the end of the follow-up were defined as clinically meaningful on the basis of the criterion of at least 0.5 standard deviations.

\section{Effect size}

The effect size is a standardized measure of change obtained by dividing the difference in scores from baseline to post-treatment by the standard deviation of baseline scores [32]. For interpreting effect sizes, Cohen [42] has proposed the following benchmarks: $0.20,0.50$, and 0.80 , indicating small, moderate, and large effects, respectively. Practically speaking, the effect size should be small in patients reporting no change and large in patients reporting a great improvement [43]. In a study by Fortin and colleagues [44], the effect size was employed to determine the responsiveness of two lupus activity measures: the revised Systemic Lupus Activity Measure (SLAM-R) and the SLEDAI. Based on this methodology, the SLAM-R performed better than or the same as the SLEDAI for both clinical improvement and worsening. With regard to no change, the two measures performed equally well.

\section{Minimal detectable change}

A measure of variability associated with the SEM is the minimal detectable change (MDC), which is the smallest detectable change that can be considered above the measurement error with a given level of confidence (usually $95 \%$ confidence) [32]. Although this method has not been used in SLE research to date, we provide an example from a study of a comprehensive rehabilitation intervention among patients with osteoarthritis by using the Western Ontario and McMaster Universities Arthritis Index (WOMAC) and the SF-36 to estimate the MDC and the MCID for improvement [45]. In the WOMAC sections, the MDC ranged from 0.75 (global) to 0.96 (stiffness), whereas in the SF-36 the MDC ranged from 2.8 (PCS) to 7.6 (physical function) [45]. The corresponding values for MCID in the WOMAC sections ranged from 0.51 to 1.33 points (on a scale of 0 to 10) and in the SF-36 the values ranged from 2.0 to 7.8 points (on a scale of 0 to 100), respectively [45]. These findings have implications for the design of meaningful clinical trials, as sections that showed moderate responsiveness (for example, the SF-36 bodily pain) require a relatively lower sample size as compared with sections that demonstrated lower responsiveness (for example, the SF-36 physical function), which require larger sample sizes. 


\section{Reliable change index}

The RCI is a statistic that assesses the magnitude of change necessary for a given self-report measure to be considered statistically reliable. It is calculated by dividing the individual patient change score by the square root of the SEM [32]. The RCI is considered to confer a true change when it is more than 1.96 (95\% confidence) (that is, the $z$-score corresponding to the desired level of significance) [32]. Although we are not aware of the application of this method in SLE research to date, it has been used to determine the clinical significance of the SF-36 [46]. Specifically, the RCIs were calculated to be 7.47 and 9.70 (reported in $\mathrm{T}$-score units, the standard metric for scoring and interpreting the SF-36), corresponding to the PCS and Mental Component Summary, respectively (calculated at the 0.05 level of significance).

\section{Standardized response mean}

The SRM is similar to the effect size, except the change in score is divided by the standard deviation of that change [23]. Similar benchmarks have been proposed to guide the interpretation of the SRM [47-49]. In the aforementioned study by Fortin and colleagues [44], the SRM was also employed to determine the responsiveness of both the SLAM- $R$ and the SLEDAI. The same pattern was noted for the comparison of the two measures as was reported for the effect size approach [44]. Furthermore, a study by Yazdany and colleagues [34] ascertained the responsiveness of the SLAQ by calculating the SRM and found that the SLAQ demonstrated a small to moderate degree of responsiveness for patients reporting a perceived change in disease status. Although the overall SRM was found to be 0.12 (that is, minimally responsive [42]), after stratification by patient changes in the patient global assessment of disease activity the SRMs were found to be 0.66 and -0.37 , corresponding to clinical deterioration and improvement, respectively. Furthermore, the SRM of no change was found to be 0.10 . These values are similar to those obtained for other commonly employed disease activity indices among patients with SLE [50].

\section{Limitations of the distribution-based methods}

First, it must be noted that the application of the various distribution-based approaches described above will result in different definitions of the MCID, which contradicts the intended aim of defining a specific threshold [32]. Most importantly, distribution-based methods are limited by their ability to define only a minimal value below which a change in outcome score for a given measure may be due to measurement error [33], which does not provide information on clinical importance. Thus, these methods largely ignore the core of the MCID, which is to define the clinical importance of a given change in outcome scores separate from their statistical significance [32].

\section{Delphi method}

The Delphi technique is a well-used method (opinionbased) for the development of a formal consensus [27, 51] and can serve as a useful adjunct to finalize MCID values following application of either the distribution- or anchorbased methods. The Delphi method involves the presentation of a questionnaire or interview to a panel of individuals in a specific field for the purpose of obtaining a consensus [52]. Participants are initially sent a questionnaire and asked to record their views; then, participants revise these responses after viewing the responses of co-participants, typically by using a Likert scale [51]. These responses are collected by the organizers and re-distributed to participating individuals as a summary of the group's judgment, as are the individuals' responses [51]. Despite substantial divergence of individual opinions in the first round of a Delphi investigation, there is a tendency for convergence of opinions toward a consensus after several iterations of this multi-step process (often repeated several times) [52]. This method has been increasingly used to develop classifications as well as response criteria for rheumatic diseases.

Recently, Brunner and colleagues [53] applied the Delphi survey method to achieve consensus on a definition of global flares in juvenile SLE and to derive candidate criteria to measure juvenile SLE flares. Outside the context of SLE, a study determining the MCID in activity limitation, fatigue, and sleep quality among rheumatoid arthritis patients initially used an internal-anchor approach, and after the preliminary MCID values were determined, the Delphi exercise was applied to reach a consensus on the final MCID values [54].

\section{Recommendations and future research agenda}

As most clinical trials in SLE to date have failed to show significant effects, determining the MCID of the instruments used to measure response may be critical for the conduct and interpretability of meaningful future clinical trials. However, little work in MCID estimation has been done in the context of SLE. Given that the MCID can help determine the effect of a given therapy on a patient and add meaning to statistical inferences made in clinical research, we believe there ought to be renewed focus on this area.

Specifically, we propose the following research agenda in the context of MCID.

1. Explicitly involving patients in defining MCIDs. Assessment of subjective yet equally important and disabling disease characteristics (for example, fatigue and physical functioning) holds the potential to incorporate the patient's perspective in a standardized way, thus facilitating the development of new therapies while making significant contributions that are valued by those who have the disease. 
2. Evaluating the patient with homogeneous levels of disease to increase responsiveness. Given the heterogeneity of SLE, we suspect that research will proceed more efficiently if some work attempts to evaluate patients with similar types of SLE (for example, individuals with a flare of lupus nephritis or with similar disease activity - either by organ involvement or global disease - level at baseline).

3. Incorporating health assessment instruments in the MCID as part of the overall assessment of response. General health assessment questionnaires, such as those that evaluate HR-QOL or use state-of-the-art methods to define a broad array of relevant symptoms (for example, the Patient Reported Outcome Measurement System, also known as PROMIS, an accessible 'item bank' to measure health concepts applicable to a variety of chronic conditions [55]) will likely be a fruitful area for future research in MCID among patients with SLE.

4. Assessment of individual organ involvement independent from overall disease activity. In addition to the assessment of overall disease activity, of particular importance is the assessment of disease outcome in terms of overall organ involvement, as SLE represents a systemic disease in which disease activity can improve in some organ systems while it worsens in others [8]. This concept is captured in the BILAG index but would benefit from adding the MCID (either alone or as part of the SRI) to further improve responsiveness, although the BILAG may have to be reweighted accordingly.

5. Development of a grading response using the MCID. Research is needed into the potential application of grading response (for example, small, moderate, or large response) in SLE using the MCID (rather than a binary concept), which may be an important discriminatory parameter to measure responsiveness in SLE.

6. Scoring using multiple instruments simultaneously. Finally, to appropriately account for these various domains in a disease as heterogeneous as SLE, it is imperative that we continue to develop a way to score multiple instruments simultaneously. To that end, further research is warranted to better understand the MCID of the various instruments available.

7. Determine whether the MCID is dependent upon the direction of the change in score (that is, clinical improvement versus worsening). As the MCID of a given self-report measure may vary depending on whether the reported change in score is positive or negative, this ought to be considered when scoring these instruments [56].
In summary, as new therapies are urgently required to treat this devastating and debilitating disease, we cannot afford to wait another 50 years before adding another therapy to the armamentarium for SLE management. Thus, to facilitate further development of new therapeutic agents for SLE, it is critical that funding agencies, researchers, patient organizations, and industry sponsors work collaboratively to close existing knowledge gaps on appropriate measurement of response in SLE.

\begin{abstract}
Abbreviations
BILAG: British Isles Lupus Assessment Group; BLISS: Belimumab in Subjects With Systemic Lupus Erythematosus; HR-QOL: health-related quality of life; MCID: minimal clinically important difference; MDC: minimal detectable change; PCS: Physical Component Summary; RCl: reliable change index; SEM: standard error of measurement; SF-36: Medical Outcomes Study 36-Item Short Form; SLAM-R: revised Systemic Lupus Activity Measure; SLAQ: Systemic Lupus Erythematosus Activity Questionnaire; SLE: systemic lupus erythematosus; SLEDAI: Systemic Lupus Erythematosus Disease Activity Index; SRI: Systemic Lupus Erythematosus Responder Index; SRM: standardized response mean; WOMAC: Western Ontario and McMaster Universities Arthritis Index.
\end{abstract}

\section{Competing interests}

The authors declare that they have no competing interests.

\section{Acknowledgments}

This work was supported by the National Institutes of Health $(\mathrm{NIH})$ under award K23AR060259. The content is solely the responsibility of the authors and does not necessarily represent the official views of the NIH. JAAZ is the BC Lupus Society Scholar. PRF holds a Tier 1 Canada Research Chair on Systemic Autoimmune Rheumatic Diseases at Université Laval.

\section{Author details}

'Arthritis Research Canada, 5591 No. 3 Road, Richmond, BC V6X 2C7, Canada. ${ }^{2}$ Department of Experimental Medicine, University of British Columbia, Vancouver General Hospital, 10226-2775 Laurel Street, Vancouver, BC V5Z 1M9, Canada. ${ }^{3}$ Department of Medicine, University of California, 1001 Potrero Ave, San Francisco, CA 94143, USA. ${ }^{4}$ Centre de recherche du CHU de Québec, Division de Rhumatologie, Département de Médecine, Université Laval, 2705 boulevard Laurier, Québec, QC G1V 2L9, Canada. ${ }^{5}$ Division of Rheumatology, Department of Medicine, University of British Columbia, Suite 802 - 1200 Burrard Street, Vancouver, BC V6Z 2C7, Canada.

\section{Published online: 03 June 2015}

\section{References}

1. Jaeschke R, Singer J, Guyatt GH. Measurement of health status. Ascertaining the minimal clinically important difference. Control Clin Trials. 1989;10:407-15.

2. Colangelo KJ, Pope JE, Peschken C. The minimally important difference for patient reported outcomes in systemic lupus erythematosus including the HAQ-DI, pain, fatigue, and SF-36. J Rheumatol. 2009;36:2231-7.

3. Yazdany J. Health-related quality of life measurement in adult systemic lupus erythematosus: Lupus Quality of Life (LupusQoL), Systemic Lupus Erythematosus-Specific Quality of Life Questionnaire (SLEQOL), and Systemic Lupus Erythematosus Quality of Life Questionnaire (L-QoL). Arthritis Care Res (Hoboken). 2011;63:S413-9.

4. Strand V, Crawford B. Improvement in health-related quality of life in patients with SLE following sustained reductions in anti-dsDNA antibodies. Expert Rev Pharmacoecon Outcomes Res. 2005;5:317-26.

5. Thumboo J, Strand V. Health-related quality of life in patients with systemic lupus erythematosus: an update. Ann Acad Med Singapore. 2007;36:115-22.

6. Leong KP, Kong KO, Thong BY, Koh ET, Lian TY, Teh CL, et al. Development and preliminary validation of a systemic lupus erythematosus-specific quality-of-life instrument (SLEQOL). Rheumatology (Oxford). 2005;44:1267-76.

7. Kuriya B, Gladman DD, Ibañez D, Urowitz MB. Quality of life over time in patients with systemic lupus erythematosus. Arthritis Rheum. 2008;59:181-5. 
8. American College of Rheumatology Ad Hoc Committee on Systemic Lupus Erythematosus Response Criteria. The American College of Rheumatology response criteria for systemic lupus erythematosus clinical trials: measures of overall disease activity. Arthritis Rheum. 2004;50:3418-26.

9. Navarra SV, Guzmán RM, Gallacher AE, Hall S, Levy RA, Jimenez RE, et al. Efficacy and safety of belimumab in patients with active systemic lupus erythematosus: a randomised, placebo-controlled, phase 3 trial. Lancet. 2011;377:721-31.

10. Furie R, Petri M, Zamani O, Cervera R, Wallace DJ, Tegzová D, et al. A phase III, randomized, placebo-controlled study of belimumab, a monoclonal antibody that inhibits B lymphocyte stimulator, in patients with systemic lupus erythematosus. Arthritis Rheum. 2011;63:3918-30.

11. Luijten KM, Tekstra J, Bijlsma JW, Bijl M. The Systemic Lupus Erythematosus Responder Index (SRI); a new SLE disease activity assessment. Autoimmun Rev. 2012;11:326-9.

12. Furie RA, Petri MA, Wallace DJ, Ginzler EM, Merrill JT, Stohl W, et al. Novel evidence-based systemic lupus erythematosus responder index. Arthritis Rheum. 2009;61:1143-51

13. Merrill JT, Neuwelt CM, Wallace DJ, Shanahan JC, Latinis KM, Oates JC, et al. Efficacy and safety of rituximab in moderately-to-severely active systemic lupus erythematosus: the randomized, double-blind, phase II/III systemic lupus erythematosus evaluation of rituximab trial. Arthritis Rheum. 2010;62:222-33.

14. Rovin BH, Furie R, Latinis K, Looney RJ, Fervenza FC, Sanchez-Guerrero J, et al. Efficacy and safety of rituximab in patients with active proliferative lupus nephritis: the Lupus Nephritis Assessment with Rituximab study. Arthritis Rheum. 2012;64:1215-26.

15. Merrill JT, Burgos-Vargas R, Westhovens R, Chalmers A, D'Cruz D, Wallace DJ, et al. The efficacy and safety of abatacept in patients with non-life-threatening manifestations of systemic lupus erythematosus: results of a twelve-month, multicenter, exploratory, phase IIb, randomized, double-blind, placebo-controlled trial. Arthritis Rheum. 2010;62:3077-87.

16. Lu TY, Jónsdóttir T, van Vollenhoven RF, Isenberg DA. Prolonged B-cell depletion following rituximab therapy in systemic lupus erythematosus: a report of two cases. Ann Rheum Dis. 2008;67:1493-4.

17. Reynolds JA, Toescu V, Yee CS, Prabu A, Situnayake D, Gordon C. Effects of rituximab on resistant SLE disease including lung involvement. Lupus. 2009;18:67-73.

18. Leandro MJ, Cambridge G, Edwards JC, Ehrenstein MR, Isenberg DA. B-cell depletion in the treatment of patients with systemic lupus erythematosus: a longitudinal analysis of 24 patients. Rheumatology (Oxford). 2005:44:1542-5.

19. van Vollenhoven RF, Gunnarsson I, Welin-Henriksson E, Sundelin B, Osterborg A, Jacobson SH, et al. Biopsy-verified response of severe lupus nephritis to treatment with rituximab (anti-CD20 monoclonal antibody) plus cyclophosphamide after biopsy-documented failure to respond to cyclophosphamide alone. Scand J Rheumatol. 2004;33:423-7.

20. Wofsy D, Hillson JL, Diamond B. Abatacept for lupus nephritis: alternative definitions of complete response support conflicting conclusions. Arthritis Rheum. 2012;64:3660-5.

21. Liang MH. Longitudinal construct validity: establishment of clinical meaning in patient evaluative instruments. Med Care. 2000;38:I|84-90

22. Beaton DE, Boers M, Wells GA. Many faces of the minimal clinically important difference (MCID): a literature review and directions for future research. Curr Opin Rheumatol. 2002;14:109-14.

23. Crosby RD, Kolotkin RL, Williams GR. Defining clinically meaningful change in health-related quality of life. J Clin Epidemiol. 2003;56:395-407.

24. Beaton DE, Bombardier C, Katz JN, Wright JG, Wells G, Boers M, et al Looking for important change/differences in studies of responsiveness. OMERACT MCID Working Group. Outcome Measures in Rheumatology. Minimal Clinically Important Difference. J Rheumatol. 2001;28:400-5.

25. Strand V, Gladman D, Isenberg D, Petri M, Smolen J, Tugwell P. Endpoints: consensus recommendations from OMERACT IV, Outcome Measures in Rheumatology. Lupus. 2000;9:322-7.

26. Smolen JS, Strand V, Cardiel M, Edworthy S, Furst D, Gladman D, et al. Randomized clinical trials and longitudinal observational studies in systemic lupus erythematosus: consensus on a preliminary core set of outcome domains. J Rheumatol. 1999;26:504-7.

27. Wells G, Beaton D, Shea B, Boers M, Simon L, Strand V, et al. Minima clinically important differences: review of methods. J Rheumatol. 2001;28:406-12.
28. Lassere MN, van der Heijde D, Johnson KR. Foundations of the minimal clinically important difference for imaging. J Rheumatol. 2001;28:890-1.

29. Lydick E, Epstein RS. Interpretation of quality of life changes. Qual Life Res. 1993;2:221-6.

30. Wyrwich KW, Bullinger M, Aaronson N, Hays RD, Patrick DL, Symonds T, et al. Estimating clinically significant differences in quality of life outcomes. Qual Life Res. 2005;14:285-95.

31. Hays RD, Woolley JM. The concept of clinically meaningful difference in health-related quality-of-life research. How meaningful is it? Pharmacoeconomics. 2000;18:419-23.

32. Copay AG, Subach BR, Glassman SD, Polly Jr DW, Schuler TC. Understanding the minimum clinically important difference: a review of concepts and methods. Spine J. 2007;7:541-6.

33. Gatchel RJ, Lurie JD, Mayer TG. Minimal clinically important difference. Spine. 2010;35:1739-43.

34. Yazdany J, Yelin EH, Panopalis P, Trupin L, Julian L, Katz PP. Validation of the systemic lupus erythematosus activity questionnaire in a large observational cohort. Arthritis Rheum. 2008:59:136-43.

35. Hudgens SA, Yost K, Cella D, Hahn EA, Peterman A. Comparing retrospective and prospective anchors for identifying minimally important differences [abstract]. Qual Life Res. 2002;11:629.

36. Wyrwich KW, Tierney WM, Wolinsky FD. Further evidence supporting an SEM-based criterion for identifying meaningful intra-individual changes in health-related quality of life. J Clin Epidemiol. 1999;52:861-73.

37. Wolinsky FD, Wan GJ, Tierney WM. Changes in the SF-36 in 12 months in a clinical sample of disadvantaged older adults. Med Care. 1998;36:1589-98.

38. McHorney CA, Tarlov AR. Individual-patient monitoring in clinical practice: are available health status surveys adequate? Qual Life Res. 1995:4:293-307.

39. Brunner HI, Higgins GC, Klein-Gitelman MS, Lapidus SK, Olson JC, Onel K, et al. Minimal clinically important differences of disease activity indices in childhood-onset systemic lupus erythematosus. Arthritis Care Res (Hoboken). 2010;62:950-9.

40. Norman GR, Sloan JA, Wyrwich KW. Interpretation of changes in health-related quality of life: the remarkable universality of half a standard deviation. Med Care. 2003;41:582-92.

41. Katz P, Morris A, Trupin L, Yazdany J, Yelin E. Disability in valued life activities among individuals with systemic lupus erythematosus. Arthritis Rheum. 2008:59:465-73.

42. Cohen J. Statistical power analysis for the behavioural sciences. New York: Academic Press; 1988.

43. Taylor SJ, Taylor AE, Foy MA, Fogg AJ. Responsiveness of common outcome measures for patients with low back pain. Spine. 1999;24:1805-12.

44. Fortin PR, Abrahamowicz M, Clarke AE, Neville C, Du Berger R, Fraenkel L, et al. Do lupus disease activity measures detect clinically important change? J Rheumatol. 2000;27:1421-8.

45. Angst F, Aeschlimann A, Stucki G. Smallest detectable and minimal clinically important differences of rehabilitation intervention with their implications for required sample sizes using WOMAC and SF-36 quality of life measurement instruments in patients with osteoarthritis of the lower extremities. Arthritis Care Res. 2001:45:384-91.

46. Ferguson RJ, Robinson $A B$, Splaine M. Use of the reliable change index to evaluate clinical significance in SF-36 outcomes. Qual Life Res. 2002;11:509-16.

47. Liang MH, Fossel AH, Larson MG. Comparisons of five health status instruments for orthopedic evaluation. Med Care. 1990;28:632-42.

48. Beaton DE, Hogg-Johnson S, Bombardier C. Evaluating changes in health status: reliability and responsiveness of five generic health status measures in workers with musculoskeletal disorders. J Clin Epidemiol. 1997;50:79-93.

49. Garratt AM, Ruta DA, Abdalla MI, Russell IT. SF 36 health survey questionnaire: II. Responsiveness to changes in health status in four common clinical conditions. Qual Health Care. 1994;3:186-92.

50. Chang E, Abrahamowicz M, Ferland D, Fortin PR, the CANIOS Investigators. Comparison of the responsiveness of lupus disease activity measures to changes in systemic lupus erythematosus activity relevant to patients and physicians. J Clin Epidemiol. 2002;55:488-97.

51. Black N, Murphy M, Lamping D, McKee M, Sanderson C, Askham J, et al. Consensus development methods: a review of best practice in creating clinical guidelines. J Health Serv Res Policy. 1999;4:236-48.

52. McKenna HP. The Delphi technique: a worthwhile research approach for nursing? J Adv Nurs. 1994;19:1221-5. 
53. Brunner HI, Klein-Gitelman MS, Higgins GC, Lapidus SK, Levy DM, Eberhard A, et al. Toward the development of criteria for global flares in juvenile systemic lupus erythematosus. Arthritis Care Res (Hoboken). 2010;62:811-20.

54. Wells $G$, Li T, Maxwell L, MacLean R, Tugwell P. Determining the minimal clinically important differences in activity, fatigue, and sleep quality in patients with rheumatoid arthritis. J Rheumatol. 2007;34:280-9.

55. Cella D, Yount S, Rothrock N, Gershon R, Cook K, Reeve B, et al. The Patient-Reported Outcomes Measurement Information System (PROMIS): progress of an NIH Roadmap cooperative group during its first two years. Med Care. 2007;45:S3-S11.

56. Cella D, Hahn EA, Dineen K. Meaningful change in cancer-specific quality of life scores: differences between improvement and worsening. Qual Life Res. 2002;11:207-21. 